\title{
Ratcheting of granular materials
}

\author{
F. Alonso-Marroquín, H. J. Herrmann \\ ICA1, University of Stuttgart, \\ Pfaffenwaldring 27, 70569 Stuttgart, Germany
}

\begin{abstract}
We investigate the quasi-static mechanical response of soils under cyclic loading using a discrete model of randomly generated convex polygons. This response exhibits a sequence of regimes, each one characterized by a linear accumulation of plastic deformation with the number of cycles. At the grain level, a quasi-periodic ratchet-like behavior is observed at the contacts, which excludes the existence of an elastic regime. The study of this slow dynamics allows to explore the role of friction in the permanent deformation of unbound granular materials supporting railroads and streets.
\end{abstract}

A particularly intriguing phenomenon in driven systems is the so-called ratchet effect. There is already an extensive body of work on this subject, driven by the need to understand biophysical systems such as molecular motors [1] and certain mechanical and electrical rectifiers [2]. The classic ratchet is a mechanical device consisting of a pawl that engages the sloping teeth of a wheel, permitting motion in one direction only. Ratchet-like motion have been proposed as a mechanism to explain the convective motion and size segregation in vibrated granular materials [3]. The understanding of this phenomenon is crucial in the investigation on the permanent deformation in structures subjected to cyclic loading. In soils, this loading can be induced by earthquakes, sea waves, road traffic, etc. [4].

The classical theory of elasto-plasticity describes the cyclic loading response by postulating an elastic region in the stress space, which changes during the deformation [5]. This elastic region, however, is not easy to identify because the onset of the plastic deformation is gradual and not sharply defined. A great variety of modifications have been proposed in order to provide a more appropriate description which, however, make the theory too complicated, and require too many material parameters which are difficult to calibrate [6].

This research was motivated by experiments of cyclic loading tests on unbound granular material used to support railroads and streets. A slow deformation is observed during the service life of these structures, where the breakage, corrosion and the friction between the grains play an important role [4]. We emphasise some recent experiments performed in Darmstadt [7]. These experiments show that when the samples consist of very wear resistant grains, the long time cyclic loading behavior is given by a linear accumulation of plastic deformation. This surprising result suggests that the grains attain a periodic irreversible motion at the sliding contacts, which could, in principle, be detected using numerical simulations. Here we report on the first micromechanical observation using molecular dynamic simulations. Ratcheting motion was detected in the sliding contacts on a polygonal packing subjected to quasi-static cyclic loading.
The polygons representing the particles in this model are generated by using a simple version of Voronoi tessellation: First, we set a random point in each cell of a regular square lattice of side $\ell$, then each polygon is constructed assigning to each point that part of the plane that is nearer to it than to any other point. This method gives a diversity of areas of polygons following a Gaussian distribution with mean value $\ell^{2}$ and variance of $0.36 \ell^{2}$. The number of edges of the polygons is distributed between 4 and 8 for $98.7 \%$ of the polygons, with a mean value of 6 .

The interaction between the polygons is modelled as follows: when two polygons overlap, two points can be defined by the intersection of their edges. The segment connecting these two intersection points defines the contact line. Then, the elastic part of the contact force is calculated as $\overrightarrow{f e}=k_{n} \Delta x_{n}^{e} \hat{n}^{c}+k_{t} \Delta x_{t}^{e} \hat{t}^{c}$, where $\hat{n}^{c}$ and $\hat{t}^{c}$ denote the normal and tangential unitary vectors with respect to the contact line, and $k_{n}$ and $k_{t}$ are the stiffnesses in the respective directions. $\Delta x_{n}^{e}$ is the overlapping area divided by a characteristic length of the interacting polygon pair [8]. $\Delta x_{t}^{e}$ defines the elastic tangential displacement of the contact, that is given by the time integral starting at the beginning of the contact

$$
\Delta x_{t}^{e}=\int_{0}^{t} v_{t}^{c}\left(t^{\prime}\right) \Theta\left(\mu f_{n}^{e}-\left|f_{t}^{e}\right|\right) d t^{\prime}
$$

where $\Theta$ is the Heaviside step function and $\vec{v}_{t}^{c}$ denotes the tangential component of the relative velocity $\vec{v}^{c}$ at the contact [9]. This equation defines a limit of elasticity in the contact force. When the contact force satisfies $f_{t}^{e}=\mu f_{n}^{e}$ the contact slides. This produces an irreversible motion between the polygons which contributes to a plastic deformation in the assembly.

In each contact a viscous force $\vec{f}^{v}=m \nu \vec{v}^{c}$ is included. Here $m$ is the relative mass of the polygons in contact and $\nu$ is the coefficient of viscosity. This force reduces the acoustic waves produced during the loading. Inertia effects were excluded by using a very long time of loading. indeed, doubling it affects the strain response by less than $5 \%$. This correspond to the quasi-static approximation. 

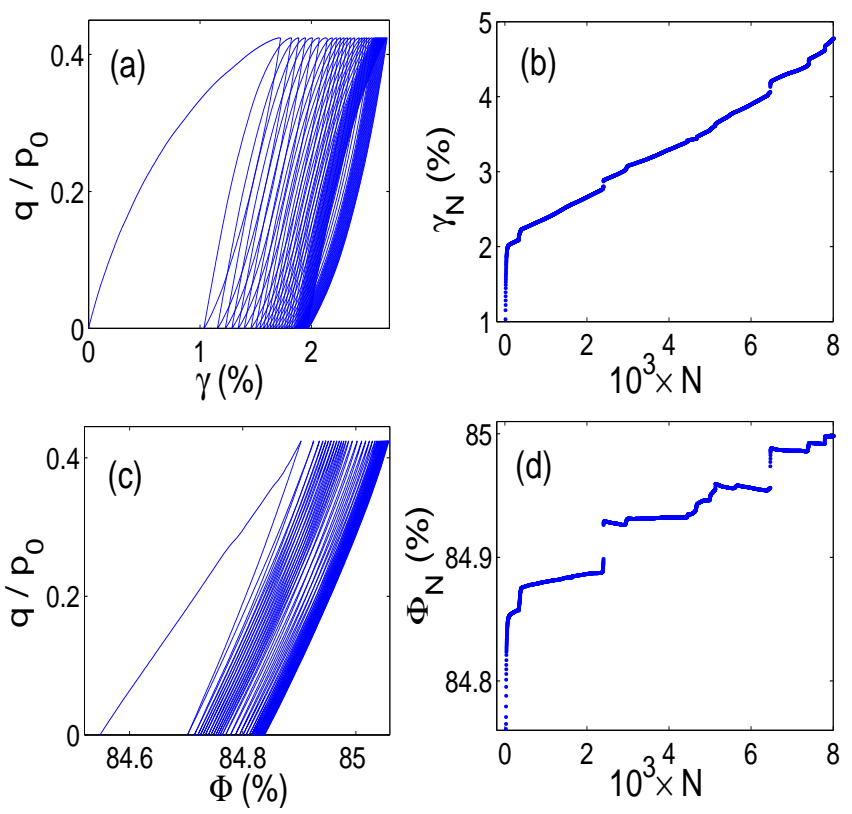

FIG. 1. (a) Shear stress versus shear strain in the first 40 cycles. (b) permanent (plastic) strain $\gamma_{N}$ after $N$ cycles versus the number of cycles. (c) stress against the volume fraction in the first 40 cycles. (d) volume fraction $\Phi_{N}$ after $N$ cycles versus number of cycles.

The parameters of our model have been reduced to a minimum set of dimensionless parameters: The period of cyclic loading $t_{0}$ is chosen 4000 times the characteristic period of oscillation $t_{s}=\sqrt{k_{n} / \rho \ell^{2}}$, where $k_{n}$ is the normal stiffness of the contacts and $\rho$ the density of the grains. The relaxation time $t_{r}=1 / \nu$ is chosen 10 times $t_{s}$. The tangential stiffness is $k_{t}=0.33 k_{n}$; the initial pressure $p_{0}=0.001 k_{n}$ and the friction coefficient $\mu=0.25$. The normal stiffness is chossen $k_{n}=160 M P a$.

The simulations are performed on six different samples of 400 polygons. First, the polygons are placed into a rectangular box such that they do not overlap each other. Next, a gravitational field is applied and the sample is allowed to consolidate. An external load is imposed by applying a force $\sigma_{1} W$ and $\sigma_{2} H$ on the horizontal and vertical walls, respectively. Here $\sigma_{1}$ and $\sigma_{2}$ are the horizontal and vertical stresses. $W$ and $H$ are the width and the height of the sample. When the velocity of the polygons vanishes gravity is switched off. A fifth-order predictor-corrector algorithm is used to solve the equations of motion.

In order to calculate the strain we select the polygons whose centers of mass are less than $10 \ell$ from the center of the sample. Then, the strain tensor is calculated as the displacement gradient tensor averaged over the area enclosed by the initial configuration of these polygons. From the eigenvalues $\epsilon_{1}$ and $\epsilon_{2}$ of the symmetric part of this tensor we obtain the shear strain as $\gamma=\epsilon_{1}-\epsilon_{2}$. The volume fraction is calculated as $\Phi=\left(V_{p}-V_{0}\right) / V_{b}$, where $V_{p}$ is the sum of the areas of the polygons, $V_{0}$ the sum of the overlapping area between them, and $V_{b}$ the area of the rectangular box. The initial volume fraction is $\Phi_{0}=84.49 \pm 0.05 \%$.

Initially, the sample is isotropically compressed until the pressure $p_{0}$ is reached. Then, the sample is subjected to vertical load-unload cycles as $\sigma_{1}=p_{0}+\frac{\sqrt{2}}{2} \Delta \sigma[1+$ $\left.\cos \left(\pi t / t_{0}\right)\right]$ whereas $\sigma_{2}=p_{0}$ is kept constant. Part (a) of Fig. 1 shows the relation between the shear stress $q=\left(\sigma_{1}+\sigma_{2}\right) / 2$ and the shear strain $\gamma$ in the case of a loading amplitude $\Delta \sigma=0.6 p_{0}$. This relation consists of open hysteresis loops which narrows as consecutive loadunload cycles are applied. This hysteresis produces an accumulation of strain with the number of cycles which is represented by $\gamma_{N}$ in the part (b) of Fig 1 . We observe that the strain response consists of short time regimes, with rapid accumulation of plastic strain, and long time ratcheting regimes, with a constant accumulation rate of plastic strain of around $2.4 \times 10^{-6}$ per cycle.

Part (c) of Fig. 1 shows the relation between the shear stress and the volume fraction. This consists of asymmetric compaction-dilation cycles which makes the sample to compact during the cyclic loading. This compaction is shown in part (d) of the Fig. 1. We observe a slow variation of the volume fraction during the ratcheting regime, and a rapid compaction during the the transition between two ratcheting regimes. The evolution of the volume ratio seems to be rather sensitive to the initial random structure of the polygons. Even so we found that after $8 \times 10^{3}$ cycles the volume fraction still slowly increasing in all the samples, without any evidence of a saturation level.

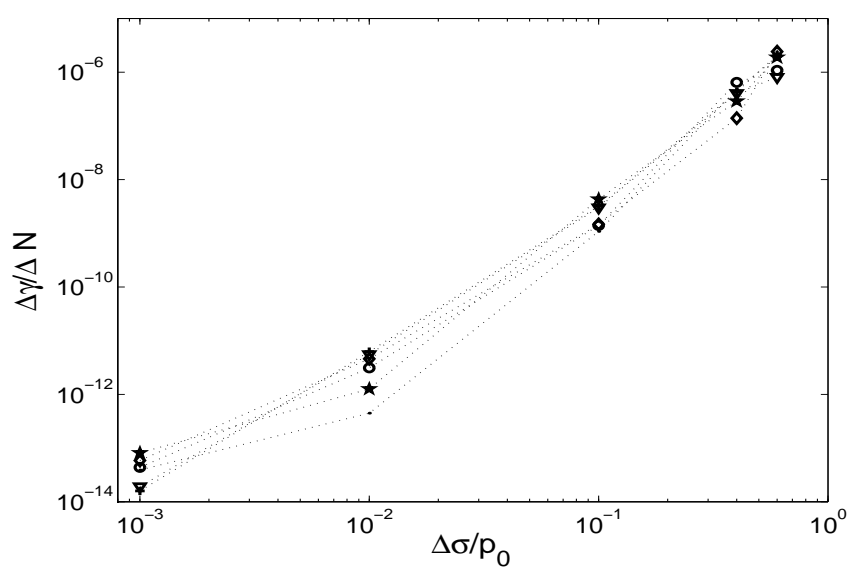

FIG. 2. Plastic deformation per cycle for different loading amplitudes. The calculations are performed on six different samples. 

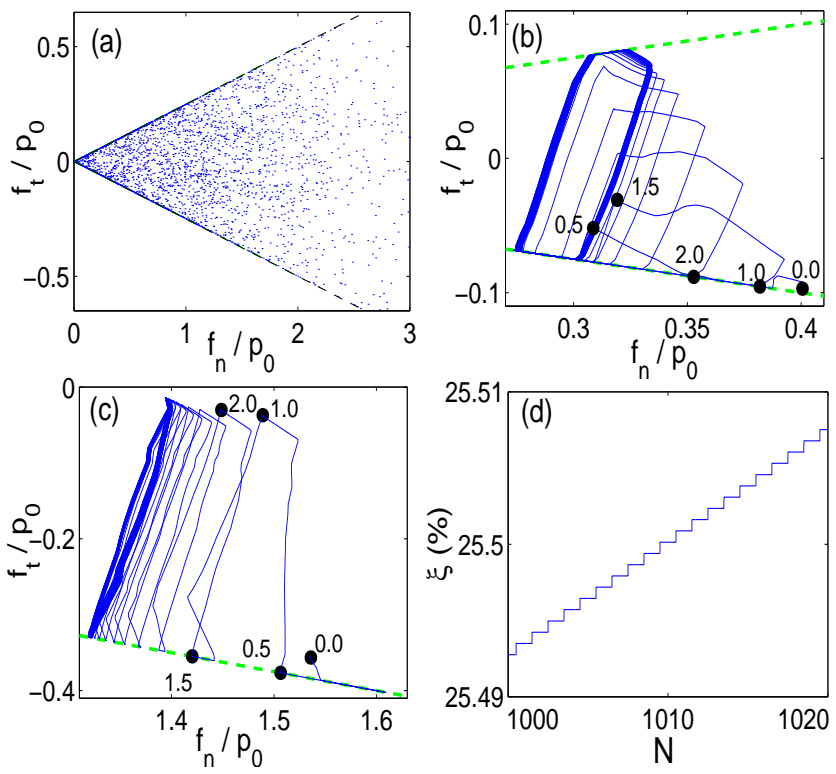

FIG. 3. (a) Each point represents the normal and tangential force at one contact. The dotted line represents the sliding condition $\left|f_{t}\right|=\mu f_{n}$. (b) and (c) Trajectories of the contact force of two selected sliding contacts. The dots denote the times $t=0,0.5 t_{0}, \ldots, 2 t_{0}$ in unit of the period $t_{0}$. (d) Plastic deformation $\xi$ at the contact shown in (c).

This extremely slow dynamics in the evolution of the granular packing shows an astonishing analogy with the behavior of glassy systems $[10,11]$. Even more surprising is that no elastic regime is detected by decreasing the amplitude of the loading cycles. Fig. 2 shows the accumulation rate of strain $\Delta \gamma / \Delta N$ for different loading amplitudes $\Delta \sigma$. A constant accumulation of strain is observed during the cyclic loading, even when the amplitude is as slow as $10^{-3}$ times the applied pressure.

The existence of these ratcheting regimes proves to be a consequence of the particular disorder of the distribution of contact forces. It is well know that the stress applied on the boundary is transmitted through an heterogeneous network of contact forces [3]. Both tangential and normal force distributions turn out to be very broad. The part (a) of Fig. 3 shows the distribution of contact forces in a polygonal packing that has been isotropically compressed. The components of the force are limited by the condition $\left|f_{t}\right| \leq \mu f_{n}$, where $\mu$ is the friction coefficient. Most of the contacts satisfy the elastic condition $\left|f_{t}\right|<\mu f_{n}$, but due to the heterogenieties, some contacts are able to reach the sliding condition $\left|f_{t}\right|=\mu f_{n}$ during the compression. Under arbitrarily small load stress cycles these contacts slide, given irreversible motion between the grains. Those sliding contacts are the most relevant micromechanic rearrangements during the cyclic loading. Opening and closure of contacts are quite rare events, and the coordination number of the packing keeps approximately it initial value $4.43 \pm 0.08$ in all the simu- lations.

After some loading cycles a quasi-periodic irreversible motion is observed at these sliding contacts. Two different sliding modes are represented in Fig. 3: (b) a contact sliding forward in the load phase and backward in the the unload one and (c) a contact sliding in the load phase and sticking in the unload one. A measure of the plastic deformation of the sliding contact is given by $\xi=\left(\Delta x_{t}^{c}-\Delta x_{t}^{e}\right) / \ell$, where $\Delta x_{t}^{c}$ and $\Delta x_{t}^{e}$ are the total and the elastic part of tangential displacement at the contact, the last one being given by Eq. 1. The part (d) of Fig. 3 shows the plastic deformation $\xi$ of the sliding contact shown in (c). Due to the load-unload asymmetry of the contact force loop, a net accumulation of plastic deformation is observed in each cycle. This is given by a slip-stick mechanism which resembles mechanical ratchets.

It is interesting to see the spatial correlation of these ratchets. Fig. 4 shows a snapshot of the field of plastic displacement per cycle at the contacts inside of the specimen. We see that correlated displacements coexist with a strongly inhomogeneous distribution of amplitudes. Localized slip zones appear periodically during each ratcheting regime. Some slip zones are destroyed and new ones are created during the transition between two ratcheting regimes. Moreover, we notice that these ratchets are found as well at the boundaries as in bulk material, without the layering effects observed in vibrated granular materials [3].

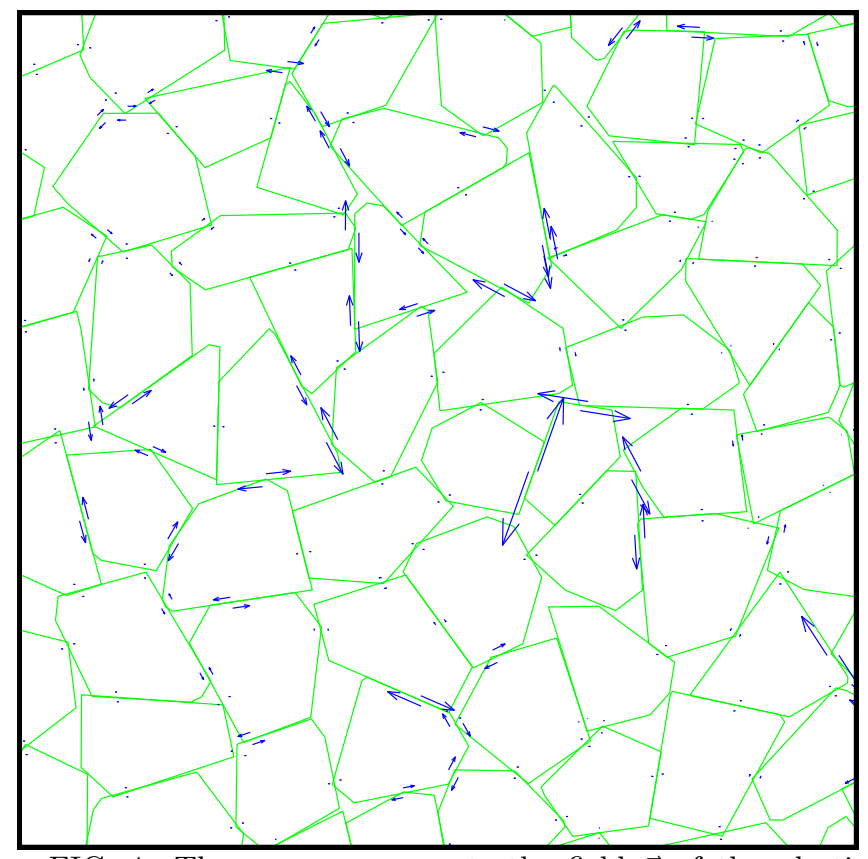

FIG. 4. The arrows represents the field $\vec{u}$ of the plastic deformations accumulated at the contacts during one cycle: $\vec{u}=500\left(\xi_{N+1}-\xi_{N}\right)$, where $\xi_{N}$ is the plastic displacement after $N$ cycles. 


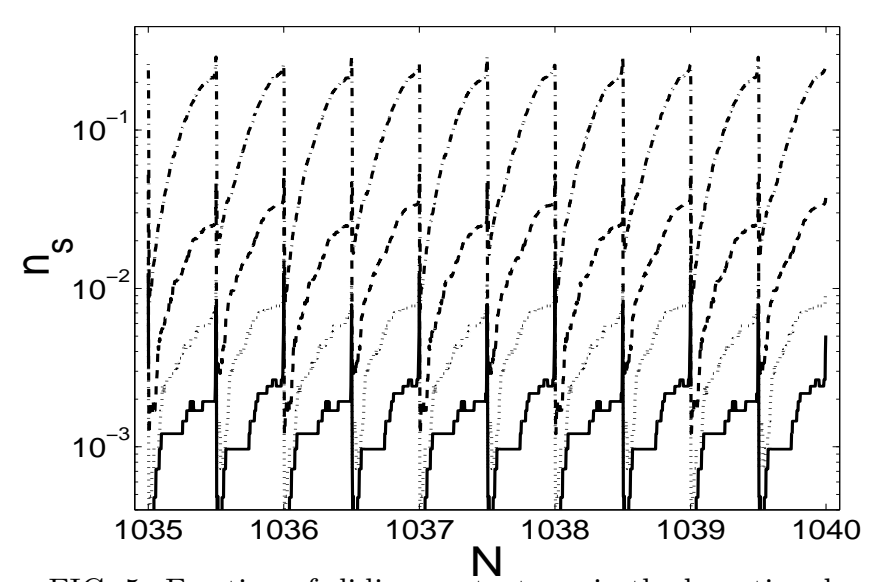

FIG. 5. Fraction of sliding contacts $n_{s}$ in the long time behavior for different values of $\Delta \sigma / p_{0}: 0.6$ (dash-dotted line), 0.1 (dashed line), 0.01 (dotted line) and 0.001 (solid line)

Let us next discuss the correlation between the dynamics of the sliding contacts and the evolution of the stiffness of the material. The last one is given by the slope of the stress strain curve in part (a) of the Fig. 1. The evolution of the fraction $n_{s}=N_{s} / N_{c}$ of sliding contacts with the number of loading cycles is shown in Fig. 5. Here $N_{s}$ is the number of sliding contacts and $N_{c}$ is the total number of contacts. During each loading phase, the number of sliding contacts increases, giving rise to a continuous decrease of the stiffness as shown in part (a) of Fig. 1. An abrupt reduction in the number of sliding contacts is observed at the transition from load to unload, producing a discontinuity in the stiffness and hence, a plastic deformation. We can see also that some contacts reach almost periodically the sliding state even for extremely small loading cycles. The ratchet-like behavior of these contacts produces a net displacement of the hysteretic stress-strain loop in each cycle, ruling out an elastic regime.

The fact that there is no evidence of an elastic regime in the cyclic loading response suggests that the hysteresis of the granular materials can not be described by using the classical theory of elasto-plasticity. Actually, the deficiencies of this theory have been addressed in the experimental investigation of soil deformation [6]. Ten years ago, the hypoplastic model was formulated in order to mend these deficiencies. This continuum theory has been supported on the experimental evidence that any load involves plastic deformation [12].

The hypoplastic approach of granular materials requires to introduce new ingredients in the current micromechanical description. The traditional fabric tensor, measuring the distribution of the orientation of the contacts, can not fulfill this description, because it does not make a distinction between elastic and sliding contacts [13]. New structure tensors taking into account the statistics of the sliding contacts, must be introduced in order to give a micro-mechanical basis to the granular ratcheting. The identification of these internal variables and the determination of their evolution equations and their connection with the macroscopic variables would be an important key to develop an appropriate continuous description of granular soils.

To conclude, we have performed a grain scale investigation of the cyclic loading response of a polygonal packing. We have shown the existence of long time regimes with a constant accumulation of plastic deformation per cycle, due to ratcheting motion at the sliding contacts. This phenomenon may have deep implications in the study of the permanent deformation of soils subjected to cyclic loading. More precisely, it may be necessary to introduce internal variables in the constitutive relations, connecting the dynamics of the sliding contacts with the evolution of the continuous variables during cyclic loading.

At this time, a comparison of the dynamic simulations with realistic situations is limited by the computer time of the simulations. Using a computer of $2.4 \mathrm{GHz}$ we are able to simulate only $3 \times 10^{3}$ cycles per week. Such amount of cycles corresponds only to some hours in realistic cases such as railroads and highways [4]. However, further investigations of the role of the stiffness, the initial stresses, and the inertial effects on this ratcheting behavior are currently feasible.

We thank G. Gudehus, P. Cundall, D. Potyondy, A. Schuenemann, J. Gallas and S. McNamara for helpful discussions and acknowledge the support of the DFG project Modellierung kohäsiver Reibungsmaterialen and the European DIGA project HPRN-CT-2002-00220.

[1] J. Howard, Nature 389, 561 (1997).

[2] P. Reimann, Phys. Rep. 61, 57 (2002).

[3] H. M. Jaeger and S. R. Nagel, Rev. Mod. Phys. 68, 1259 (1996).

[4] F. Lekarp, A. Dawson, and U. Isacsson, J. Transp. Engrg. 126, 76 (2000).

[5] D. Drucker and W. Prager, Q. Appl. Math. 10, 157 (1952).

[6] Constitutive Relations of soils, edited by G. Gudehus, F. Darve, and I. Vardoulakis (Balkema, Rotterdam, 1984).

[7] G. Festag, dissertation TU Darmstadt (unpublished).

[8] F. Kun and H. J. Herrmann, Phys. Rev. E 59, 2623 (1999).

[9] F. Alonso-Marroquin and H. J. Herrmann, Phys. Rev. E 66, 021301 (2002), cond-mat/0203476.

[10] A. J. Liu and S. Nagel, Nature 296, 21 (1998).

[11] P. D. M. Nicolas and O. Pouliquen, Eur. Phys. J. E 3, 309 (2000).

[12] D. Kolymbas, Arch. of Appl. Mech. 61, 143 (1991).

[13] R. Bathurst and L. Rothenburg, J. Appl. Mech. 55, 17 (1988). 